\title{
Performance evaluation of quarter shift dual tree complex wavelet transform based multifocus image fusion using fusion rules
}

\author{
N. Radha', T. Ranga Babu' \\ ${ }^{1,3}$ Department of Electronics and Communication Engineering, Acharya Nagarjuna University, India \\ ${ }^{2}$ Department of ECE, RVR and JC College of Engineering, India
}

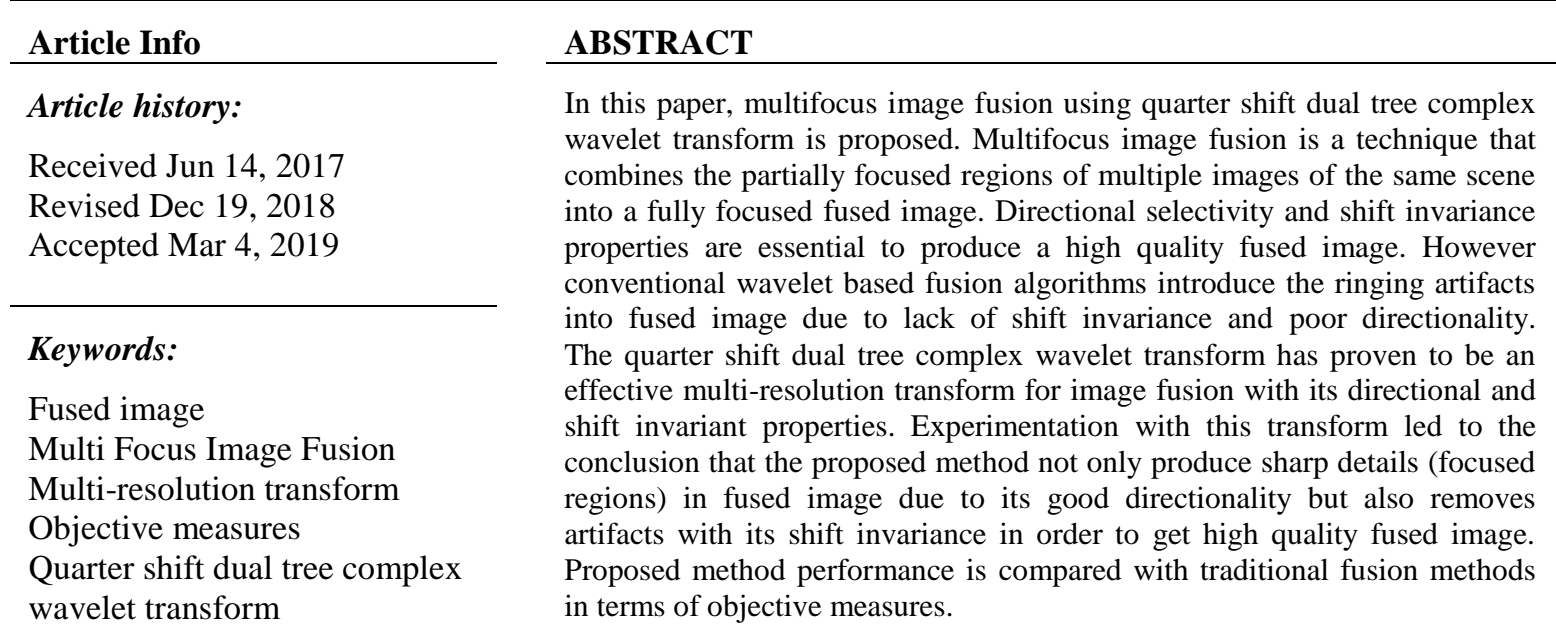

Copyright () 2019 Institute of Advanced Engineering and Science. All rights reserved.

\section{Corresponding Author:}

N. Radha,

Department of Electronics and Communication Engineering,

Acharya Nagarjuna University,

Guntur, Andhra Pradesh, India.

Email: radha_naina@yahoo.com

\section{INTRODUCTION}

The derivation of an image that comprises all relevant objects in focus became impossible due to the restricted depth of focus of optical lenses in CCD devices [1]. The solution to this problem is multi-focus image fusion, which combines multiple images of the same scene into a composite image which is more feasible for visualization and detection [2].The multi-focus image fusion methods are spatial and transform domain methods [3].Transform domain algorithms namely the multi-resolution algorithms, are more robust since the human visual system deals with information in a multi-resolution way, which is in line with the processing principle of transform domain algorithms.

In literature various multi-resolution techniques are developed like the laplacian pyramid [4], gradient pyramid [5], discrete wavelet transform (DWT) [6], stationary wavelet transform (SWT) [7-9], multi-resolution singular value decomposition (MSVD) [10], discrete cosine harmonic wavelet transform (DCHWT) [11], lifting wavelet transform[12-13], double density discrete wavelet transform (DDDWT) [14] and Shearlet Transform [15]. The major problem with pyramid based methods is lack of spatial orientation selectivity, which results in blocking effect in the fused image. Use of DWT can prevent this pitfall. But DWT suffers from aliasing, dearth of shift invariance and directionality. Shift invariance and directional selectivity are primary to fused images quality. The conventional wavelet based fusion algorithms introduce the ringing artifacts into fused image, which limit the use of DWT for image fusion. 
The dual tree complex wavelet transform (DTCWT) [16] is one of the most precise ones eliminating dearth of shift invariance and directional sensitivity caused by DWT.DTCWT, being of near shift invariance and better directional selectivity, can well represent details in fused image. However, in DTCWT, the process of designing filters is a little complicated due to its requirement for satisfying both bi-orthogonal and phase conditions. The quarter shift dual tree complex wavelet transform (q shift DTCWT) is a solution to simplify the construction of filters in DTCWT, producing better fusion results. The q shift DTCWT has verified to be an effective multi-resolution transform for image fusion with its ability to pick up the directional and shift invariant properties.

\section{QUARTER SHIFT DUAL TREE COMPLEX WAVELET TRANSFORM}

The DWT that is intensely sampled suffers from a dearth of shift invariance in 1-D and directional sensitivity in N-D. To conquer these problems, DTCWT, which is approximately shift-invariant, computationally efficient and directionally selective, is developed. The DTCWT is an advanced wavelet transform which produces the real and imaginary parts of transform coefficients by using a dual-tree of real wavelet filters. Implementation of the DTCWT is done with two different two-channel FIR filter banks. For one of the filter banks the output is considered to be the real part (Tree A), while for the other filter bank the output is considered to be the imaginary part (Tree B). Two critically sampled filter banks are used by the DTCWT; for a d-dimensional image the redundancy is $2^{\mathrm{d}}$. Figure 1 reflects three levels of the filter bank structure for 1-D DTCWT.

Due to its shift invariance, images fused by DTCWT are smooth and continuous while images fused by DWT contain irregular edges. Another major superiority of DTCWT is good directional selectivity since DTCWT produces six sub-bands for both real and imaginary parts in $( \pm 15 \circ, \pm 45 \circ, \pm 75 \circ)$ at each scale, whereas DWT merely presents limited directions in $(0 \circ, 45 \circ, 90 \circ)$, which improves the transform precision and keeps more detailed information.

However, there exist a few problems with the odd/even filter approach in DTCWT [16]:

i. Good symmetry doesn't exist in the sub-sampling structure

ii. Frequency responses are slightly different for the two trees;

iii. The filter sets need to be bi-orthogonal, rather than being orthogonal, as they are linear phase.

This reflects that preservation of energy doesn't exist between the signals and transform domains.

To minimize and overcome all of the above, a q shift DTCWT is proposed, as in Figure 2, where one can notice even length in all the filters beyond level 1.

Beyond level 1, 1/2 sample delay difference is obtained with filter delays of 1/4 and 3/4 of a sample period (instead of 0 and 1/2 a sample for our original DTCWT). An asymmetric even-length filter and its time reverse can achieve this. Owing to the asymmetry, these can be designed to produce an orthonormal perfect reconstruction wavelet transform. Reverse of Tree-A filters are Tree-B filters, and reverse of analysis filters are reconstruction filters, so all filters are from the same orthonormal set. Same frequency responses are reflected in both the trees. Though the separate responses are asymmetric, the combined complex impulse responses are conjugated symmetric about their mid points. It is for this reason that symmetric extension still works at image edges.

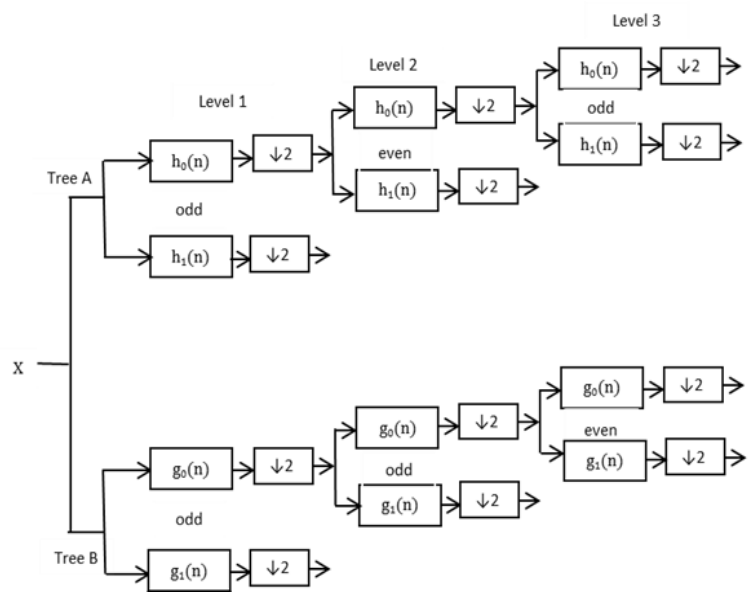

Figure 1. Filter bank structure for DTCWT

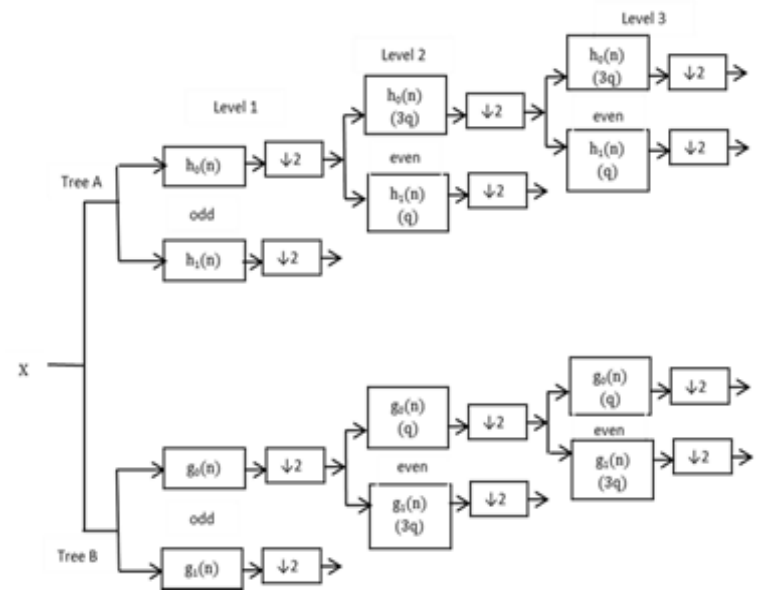

Figure 2. Q shift DTCWT filter structure 


\section{PROPOSED METHOD}

When two input images A and B are considered, the proposed method contains the following steps:

a. Perform $l$-level q shift DTCWT on source images A and B to get low and high-frequency sub- bands at each level $l$ and direction $d$ denoted as in (1)

$$
\mathrm{A}:\left\{\mathrm{L}_{l}^{\mathrm{A}}, \mathrm{H}_{l, d}^{\mathrm{A}}\right\}, \text { and } \quad \mathrm{B}:\left\{\mathrm{L}_{l}^{\mathrm{B}}, \mathrm{H}_{l, d}^{\mathrm{B}}\right\}
$$

Where $\mathrm{L}_{l}^{\mathrm{A}}, \mathrm{L}_{l}^{\mathrm{B}}$ : low frequency coefficients

$$
\mathrm{H}_{l, d}^{\mathrm{A}}, \mathrm{H}_{l, d}^{\mathrm{B}}: \text { high frequency coefficients }
$$

b. Fusion of low- frequency sub- bands: Shannon entropy based fusion rule is used to fuse low frequency coefficients in low- frequency sub- bands using (2)-(6).

1) Shannon entropy of low-frequency coefficients in a region $R$ centered at $(x, y)$ is calculated as

$$
\begin{aligned}
& E_{A}(x, y)=\sum_{i, j \in R} L_{l}^{A}(i, j)^{2} \log \left(L_{l}^{A}(i, j)^{2}\right) \\
& E_{B}(x, y)=\sum_{i, j \in R} L_{l}^{B}(i, j)^{2} \log \left(L_{l}^{B}(i, j)^{2}\right)
\end{aligned}
$$

2) Salient information is extracted from each image low-frequency coefficients at location $(x, y)$ as

$$
\begin{aligned}
& S_{A}(x, y)=\frac{E_{A}(x, y)}{E_{A}(x, y)+E_{B}(x, y)} \\
& S_{B}(x, y)=\frac{E_{B}(x, y)}{E_{A}(x, y)+E_{B}(x, y)}
\end{aligned}
$$

3) Low-frequency coefficients are fused as

$$
\mathrm{L}_{l}^{\mathrm{F}}(\mathrm{x}, \mathrm{y})=\mathrm{S}_{\mathrm{A}}(\mathrm{x}, \mathrm{y}) \mathrm{L}_{l}^{\mathrm{A}}(\mathrm{x}, \mathrm{y})+\mathrm{S}_{\mathrm{B}}(\mathrm{x}, \mathrm{y}) \mathrm{L}_{l}^{\mathrm{B}}(\mathrm{x}, \mathrm{y})
$$

c. Fusion of high-frequency sub-bands: high-frequency coefficients in high-frequency sub- bands are fused based on fusion rule using (7), to select the larger absolute value of the high-frequency coefficients, since these coefficients correspond to edges and object boundaries etc.

$$
\mathrm{H}_{l, d}^{\mathrm{F}}(\mathrm{x}, \mathrm{y})= \begin{cases}\mathrm{H}_{l, d}^{\mathrm{A}}(\mathrm{x}, \mathrm{y}), & \text { if }\left|\mathrm{H}_{l, d}^{\mathrm{A}}(\mathrm{x}, \mathrm{y})\right| \geq\left|\mathrm{H}_{l, d}^{\mathrm{B}}(\mathrm{x}, \mathrm{y})\right| \\ \mathrm{H}_{l, d}^{\mathrm{B}}(\mathrm{x}, \mathrm{y}), & \text { if }\left|\mathrm{H}_{l, d}^{\mathrm{A}}(\mathrm{x}, \mathrm{y})\right|<\left|\mathrm{H}_{l, d}^{\mathrm{B}}(\mathrm{x}, \mathrm{y})\right|\end{cases}
$$

d. Perform $l$-level inverse q shift DTCWT on the composited low- and high-frequency sub-bands to obtain the fused image.

\section{EVALUATION} measures.

The proposed method's performance can be evaluated using reference and non-reference objective

\subsection{Reference measures}

a. Peak Signal to Noise Ratio (PSNR): The PSNR between the ground truth image R and the fused image $\mathrm{F}$ is defined as in (8)

$$
P S N R=10 \log _{10}\left[\frac{P_{\max } \times P_{\max }}{\frac{1}{N^{2}} \sum_{i, j=1}^{N}[R(x, y)-F(x, y)]^{2}}\right]
$$

b. Structural Similarity Index Measure (SSIM) [17]: Representation of SSIM between the ground truth image $\mathrm{R}$ and fused image $\mathrm{F}$ is given by (9)

$$
\operatorname{SSIM}=\frac{\left(2 \mu_{R} \mu_{F}+C_{1}\right)\left(2 \sigma_{R F}+C_{2}\right)}{\left(\mu_{R}^{2}+\mu_{F}^{2}+C_{1}\right)\left(\sigma_{R}^{2}+\sigma_{F}^{2}+C_{2}\right)}
$$




\subsection{Non-reference measures}

a. Standard deviation: It is used to evaluate the fused image contrast. Which is defined as (10):

$$
\sigma=\sqrt{\sum_{\mathrm{i}=0}^{\mathrm{L}}(\mathrm{i}-\overline{\mathrm{l}})^{2}} \mathrm{~h}_{\mathrm{I}_{\mathrm{f}}}(\mathrm{i}), \overline{\mathrm{l}}=\sum_{\mathrm{i}=0}^{\mathrm{L}} \mathrm{ih}_{\mathrm{I}_{\mathrm{f}}}
$$

b. Spatial Frequency (SF): To quantity the clarity level of an image, spatial frequency can be used. If the SF value is larger it denotes better fusion result and is defined using (11)-(13):

$$
\begin{aligned}
& \mathrm{RF}=\sqrt{\frac{1}{\mathrm{MN}} \sum_{\mathrm{x}=1}^{\mathrm{M}} \sum_{\mathrm{y}=2}^{\mathrm{N}}\left[\mathrm{I}_{\mathrm{F}}(\mathrm{x}, \mathrm{y})-\mathrm{I}_{\mathrm{F}}(\mathrm{x}, \mathrm{y}-1)\right]^{2}} \\
& \mathrm{CF}=\sqrt{\frac{1}{\mathrm{MN}} \sum_{\mathrm{x}=1}^{\mathrm{M}} \sum_{\mathrm{y}=2}^{N}\left[\mathrm{I}_{\mathrm{F}}(\mathrm{x}, \mathrm{y})-\mathrm{I}_{\mathrm{F}}(\mathrm{x}-1, \mathrm{y})\right]^{2}} \\
& \mathrm{SF}=\sqrt{\mathrm{RF}^{2}+\mathrm{CF} \mathrm{F}^{2}}
\end{aligned}
$$

\section{RESULTS AND ANALYSIS}

The proposed method is verified on source images of multi-focus reference images, non-reference images and microscopic images.

\subsection{Experiment on multifocus images (with reference image)}

The first experiment is performed using artificially created source images with divergent focus levels. Three standard images Cameraman, Boat, Lena of USC-SIPI database are used as reference images in this experiment. For each reference image, two source images are produced by filtering the reference image with a $5^{\times 5}$ gaussian filter centered at the top, bottom, and left and right parts respectivly. Both the reference and artificially generated source images are shown in Figures 3, 4 and 5.

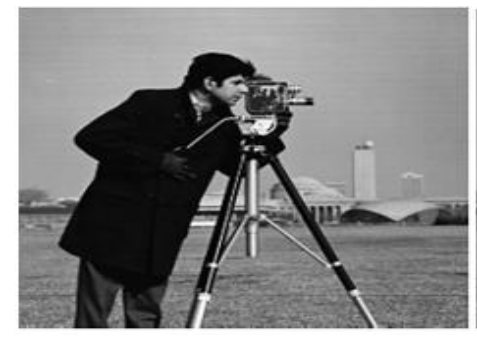

(a)

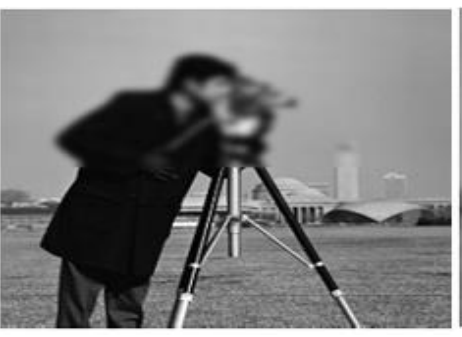

(b)

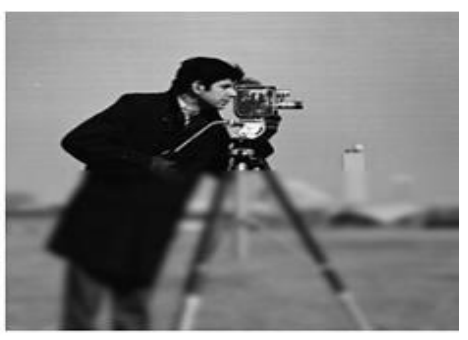

(c)

Figure 3. Reference and source images of Cameraman, (a) Reference image, (b) First source image with blur on top, (c) Second source image with blur on bottom

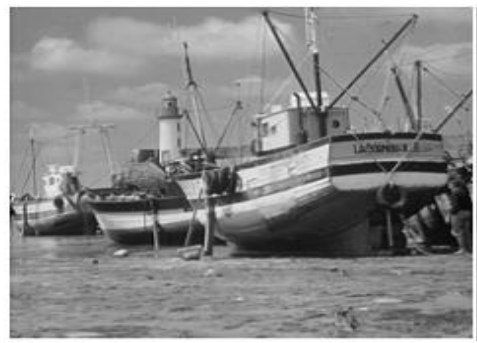

(a)

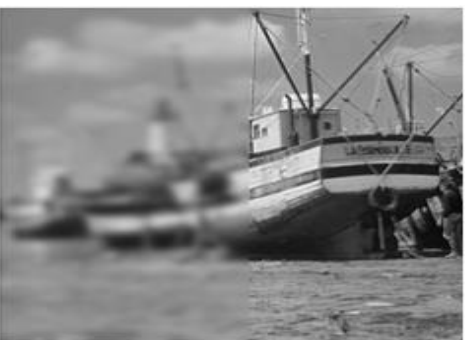

(b)

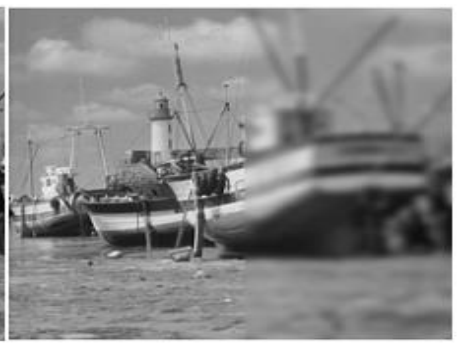

(c)

Figure 4. Reference and source images of Boat, (a) Reference image,

(b) First source image with blur on left, (c) Second source image with blur on right 


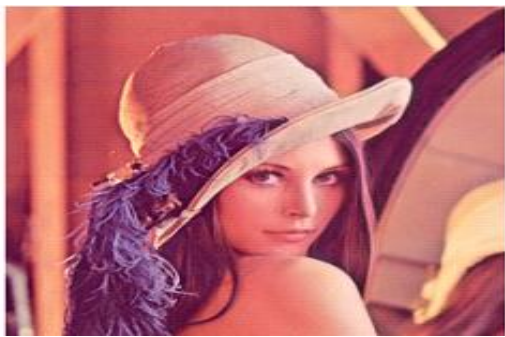

(a)

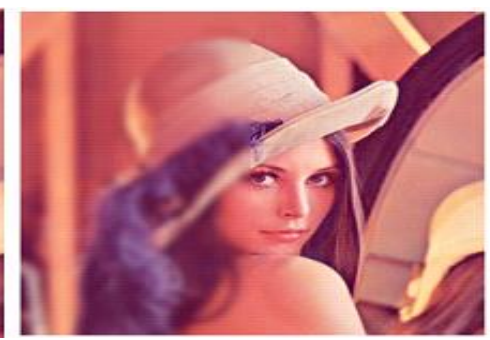

(b)

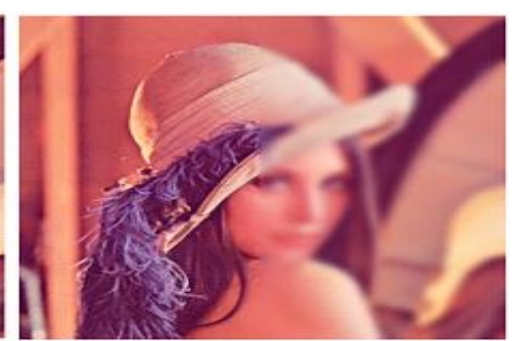

(c)

Figure 5. Reference and source images of Lena, (a) Reference image,

(b) First source image with blur on left, (c) Second source image with blur on right

The qualitative analysis of proposed method has been done using fusion results of cameraman image by different fusion methods as shown in Figure 6(a)-(f). From Figure 6(a), one can find that DWT method [6], yields ringing artifacts in fused image due to lack of shift-invariance. However, fused image of SWT method [7], in Figure 6(b) reduces the contrast in fused image. The MSVD method [10], in Figure 6(c) shows discontinuities in edges. The DCHWT method [11], introduces blocking artifacts in fused image in Figure 6(d). The DDDWT method [14], also yields blurring and ringing artifacts in fused image shown in Figure 6(e). One can observe from Figure 6(f) that the proposed method gives fused image without blurring and good contrast. The logic is that the shift-invariance property of q-shift DTCWT in proposed method reduces artifacts with perfect reconstructed fused image and its directional selectivity represents edges more efficiently. Similar observations are found in experimentation with boat and Lena images as shown in Figures 7 and 8. The quantitative analysis of proposed method is done by comparing the fused image with reference image through reference measures like PSNR and SSIM as given in Table 1. From Table 1 it can be observed that, PSNR and SSIM values obtained in the proposed method are better than any other fusion methods.

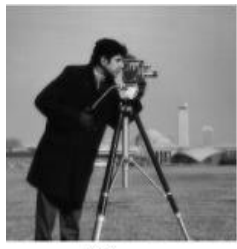

(a)

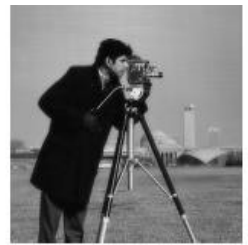

(b)

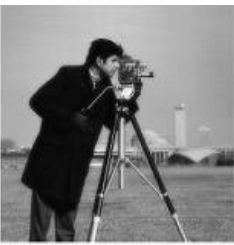

(c)

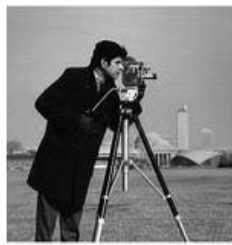

(d)

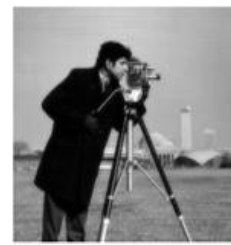

(e)

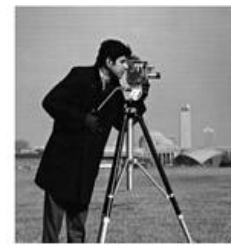

(f)

Figure 6. Comparison of fusion results of different fusion methods (Cameraman) (a) Fused image using DWT [6], (b) Fused image using SWT [7], (c) Fused image using MSVD [10], (d) Fused image using

DCHWT [11], (e) Fused image using DDDWT [14], (f) Fused image using proposed method

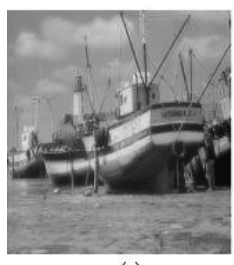

(a)

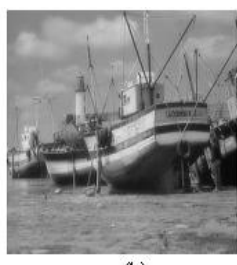

(b)

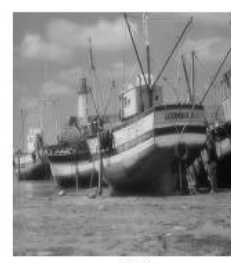

(c)

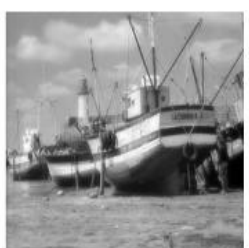

(d)

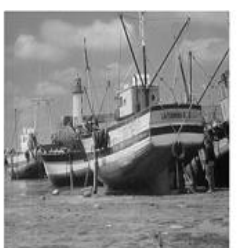

(e)

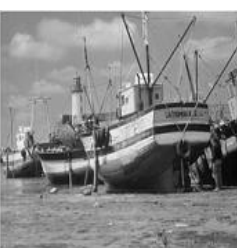

(f)

Figure 7. Comparison of fusion results of different fusion methods (Boat) (a) Fused image using DWT [6], (b) Fused image using SWT [7], (c) Fused image using MSVD [10], (d) Fused image using DCHWT [11],

(e) Fused image using DDDWT [14], (f) Fused image using proposed method 


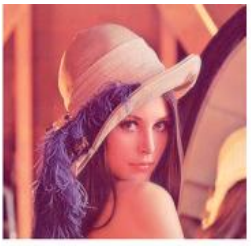

(a)

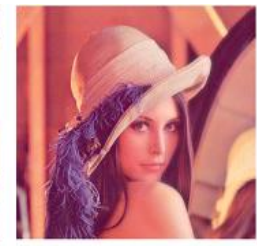

(b)

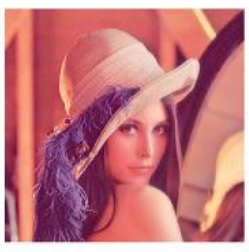

(c)

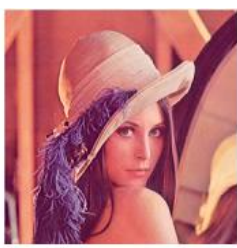

(d)

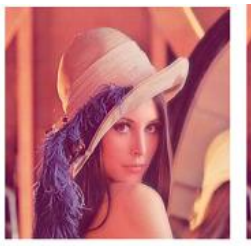

(e)

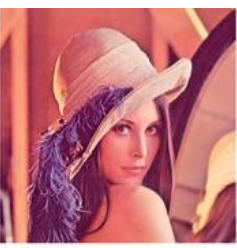

Figure 8. Comparison of fusion results of different fusion methods (Lena) (a) Fused image using DWT [6], (b) Fused image using SWT [7], (c) Fused image using MSVD [10], (d) Fused image using DCHWT [11],

(e) Fused image using DDDWT [14], (f) Fused image using proposed method.

Table 1. Comparison of reference measures of proposed method with different fusion methods

\begin{tabular}{lcccccc}
\hline Fusion method & \multicolumn{2}{c}{ Cameraman } & \multicolumn{2}{c}{ Test image } & \multicolumn{2}{c}{ Boat } \\
& PSNR & SSIM & PSNR & SSIM & PSNR & SSIM \\
\hline DWT [6] & 27.4231 & 0.8903 & 27.9141 & 0.8758 & 29.9345 & 0.9814 \\
SWT [7] & 30.8535 & 0.9643 & 31.9237 & 0.9631 & 32.1914 & 0.9896 \\
MSVD [10] & 29.2801 & 0.8642 & 30.7809 & 0.9186 & 31.3313 & 0.9868 \\
DCHWT [11] & 40.0761 & 0.9076 & 40.6855 & 0.9690 & 36.3184 & 0.9953 \\
DDDWT [14] & 28.6193 & 0.8159 & 29.1821 & 0.8608 & 32.5541 & 0.9910 \\
Proposed method & 44.3526 & 0.9927 & 44.1491 & 0.9947 & 36.8943 & 0.9957 \\
\hline
\end{tabular}

\subsection{Experiment on multifocus real images (with out reference image)}

The second experiment is run using two sets of multifocus images with out reference image: Pepsi and Clock, as presented in Figures 9 and 10 respectively. The qualitative analysis of proposed method has been done using fusion results of Pepsi and Clock image are shown in Figures 11 and 12. The use of average fusion rule for selection of low frequency components in DWT, SWT and MSVD methods introduce artifacts, contrast reduction and edge discontinuities in pepsi fused image as shown in Figure 11(a)-(c). The DCHWT method, introduces blocking artifacts in fused image in Figure 11(d) due to weighted average fusion rule. However, absolute maximum fusion rule for fusion of low frequency components in DDDWT method yields blurring shown in Figure 11(e). One can observe from Figure 11(f) that the proposed method preserves edges and produce good contrast in fused image. The logic is that the use of shannon entropy fusion rule selects low frequency components from the focused regions and the use of larger absolute value fusion rule selects high-frequency coefficients. Similar observations are found in experimentation with clock image as shown in Figure 12 (a)-(f). The quantitative analysis of proposed method on multifocus nonreference images is done through non-reference measures like SD and SF as given in Table 2. It is found from Table 2 that the proposed method results high SD and SF values than other fusion methods. Hence, the proposed method well transfers sharp details from source images to fused image.
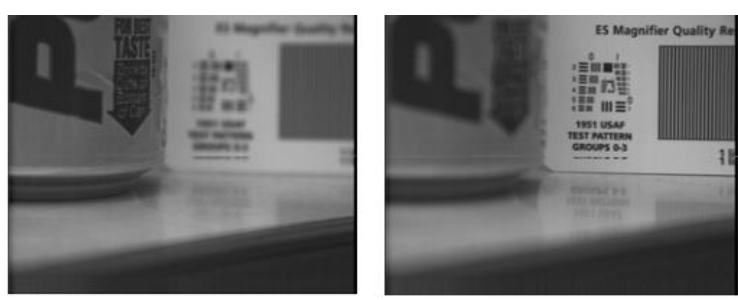

Figure 9. Two source images of Pepsi image,

(a) First source image with focused foreground, (b) Second source image with focused background
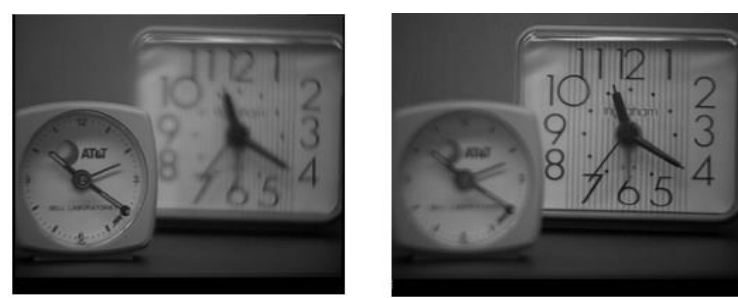

Figure 10. Two source images of Clock image, (a) First source image with focused foreground, (b) Second source image with focused background 


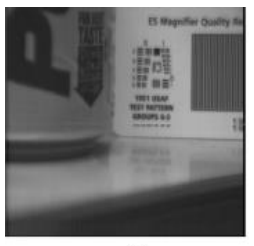

(a)

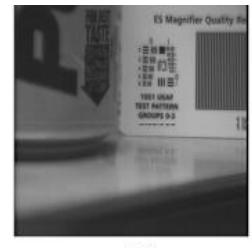

(b)

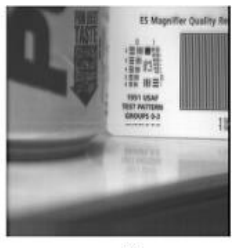

(c)

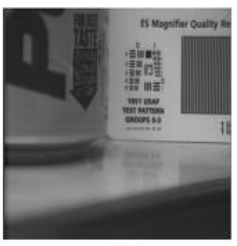

(d)

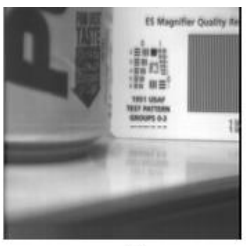

(e)

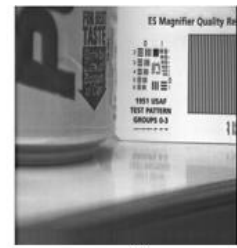

(f)

Figure 11. Comparison of fusion results of different fusion methods (Pepsi) (a) Fused image using DWT [6], (b) Fused image using SWT [7], (c) Fused image using MSVD [10], (d) Fused image using DCHWT [11], (e) Fused image using DDDWT [14], (f) Fused image using proposed method

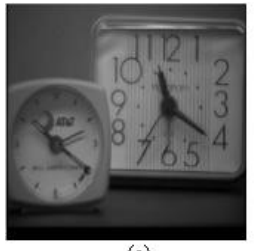

(a)

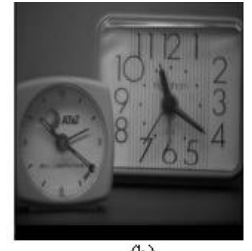

(b)

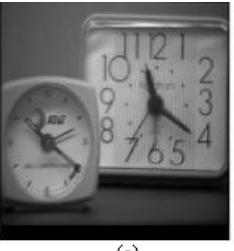

(c)

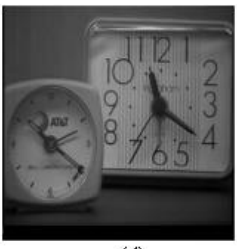

(d)

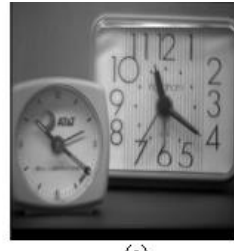

(e)

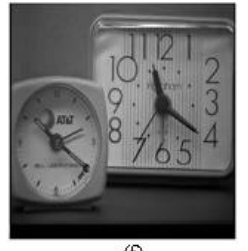

(f)

Figure 12. Comparison of fusion results of different fusion methods (Clock) (a) Fused image using DWT [6], (b) Fused image using SWT [7], (c) Fused image using MSVD [10], (d) Fused image using DCHWT [11],

(e) Fused image using DDDWT [14], (f) Fused image using proposed method

Table 2. Comparison of non-reference measures of proposed method with different fusion methods

\begin{tabular}{lcccccc}
\hline Fusion method & \multicolumn{2}{c}{ Pepsi } & \multicolumn{2}{c}{ Test image } & \multicolumn{2}{c}{ Microscope } \\
& SD & SF & SD & SF & SD & SF \\
\hline DWT [6] & 43.9368 & 13.7941 & 39.5437 & 9.0516 & 18.0925 & 8.8698 \\
SWT [7] & 43.9723 & 13.3940 & 39.8556 & 10.9479 & 18.6411 & 11.1288 \\
MSVD [10] & 44.0172 & 13.8258 & 39.6455 & 11.2043 & 18.7129 & 11.5933 \\
DCHWT [11] & 44.3854 & 14.8529 & 40.3674 & 12.2549 & 19.2598 & 12.7251 \\
DDDWT [14] & 43.5475 & 9.6315 & 39.3944 & 8.8223 & 18.0099 & 8.0941 \\
Proposed method & 44.9180 & 15.5403 & 41.1259 & 13.7913 & 19.7708 & 13.3553 \\
\hline
\end{tabular}

\subsection{Experiment on multifocus microscopic images}

In third experiment, the proposed method is also verified on multi-focus microscope images as presented in Figure 13. Due to limited depth of field of microscope, it is not possible to generate all objects in focus in microscope image. The fusion results of microscopic image are shown in Figure 14 (a)-(f). From Figure 14 (f) one can notice that the proposed method can well combine sharp features from source images into the fused image. The comparison of quantitative evaluation of different fusion methods for microscopic image is given in Table 2. SD and SF values obtained in the proposed method are better than any other fusion methods.

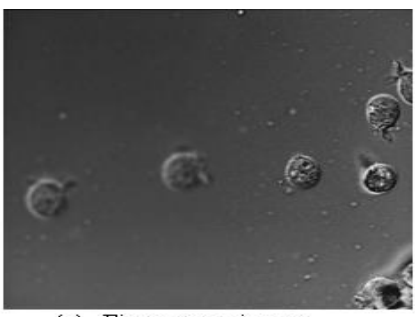

(a) First source image
with focus on righ

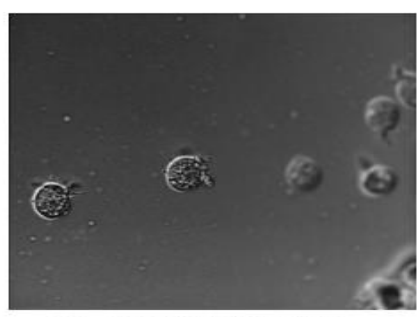

(b) Second source image
with focus on left

Figure 13. Microscopic source images, (a) First source image with focus on right,

(b) Second source image with focus on left 


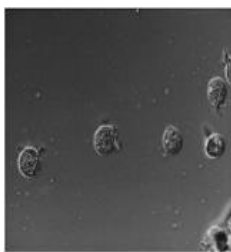

(a)

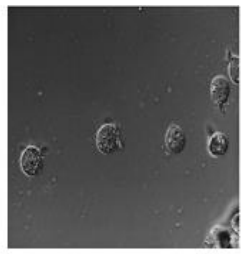

(b)

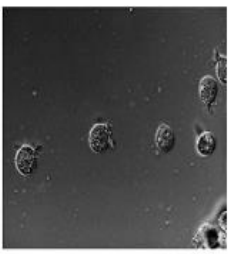

(c)

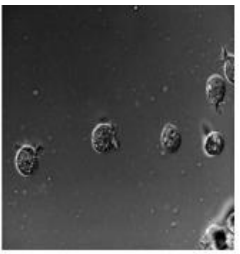

(d)

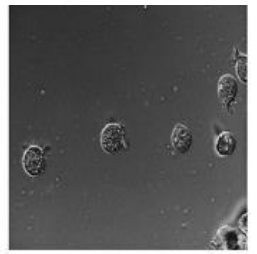

(e)

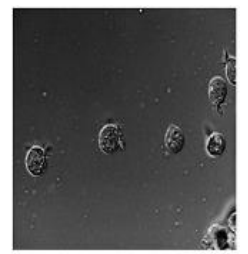

(f)

Figure 14. Comparison of fusion results of different fusion methods (microscopic image), (a) Fused image using DWT [6], (b) Fused image using SWT [7], (c) Fused image using MSVD [10], (d) Fused image using DCHWT [11], (e) Fused image using DDDWT [14], (f) Fused image using proposed method

\section{CONCLUSION}

In this paper, multi-focus image fusion using quarter shift dual tree complex wavelet transform is proposed. Good directionality, high degree of shift-invariance, phase information and computational efficiency of q shift DTCWT make the algorithm suitable for image fusion and help to produce a high quality fused image. And also the fusion rules helps to get focused fused image. Experimental results demonstrate that the proposed method preserves the image details in a far better way and extensively improves the image sharpness compared to the other fusion methods with very less information loss. The proposed fusion method is tested on both multi-focus and microscopic images. This indicates that it is well suited for real time applications.

\section{REFERENCES}

[1] P. Shah, SN. Merchant, and UB. Desai, "Multifocus and multispectral image fusion based on pixel significance using multiresolution decomposition," Signal Image and Video Processing, vol.7, pp. 95-109, 2013.

[2] Y. Chai, H. Li, and Z. Li, "Multifocus image fusion scheme using focused region detection and multiresolution," Optics Communications, vol. 284, pp. 4376-4389, 2011.

[3] B. Zhang, C. Zhang, L. Yuanyuan, W. Jianshuai, and L. He, "Multi-focus image fusion algorithm based on compound PCNN in Surfacelet domain," Optik 125, pp. 296-300, 2014.

[4] I.S. Wahyuni and R. Sabre, "Wavelet Decomposition in Laplacian Pyramid for Image Fusion," International Journal of Signal Processing Systems, vol. 4, pp. 37-44, 2016.

[5] V. Petrovic and C. Xydeas, "Gradient-based multiresolution image fusion," IEEE Transactions Image Processing, vol.13, pp. 228-237, 2004.

[6] W.W. Wang, P. Shui, and G. Song, "Multifocus Image Fusion In Wavelet Domain," in Second International Conference on Machine Learning and Cybernetics,2003.IEEE,2003, pp. 2887-2890.

[7] S. Li, B.Yang, and J. Hu, "Performance comparison of different multi-resolution transforms for image fusion," Information Fusion, vol.12, pp.74-84, 2011.

[8] Abhishek Sharma and Tarun Gulati, "Change Detection from Remotely Sensed Images Based on Stationary Wavelet Transform," International Journal of Electrical and Computer Engineering, vol. 7, No. 6, pp. 3395-3401, 2017.

[9] P. Borwonwatanadelok, W. Rattanapitak, and S. Udomhunsakul, "Multi-Focus Image Fusion based on Stationary Wavelet Transform and extended Spatial Frequency Measurement," in International Conference on Electronic Computer Technology, 2009.IEEE, pp. 77-81, 2009.

[10] V.P.S. Naidu, "Image Fusion Technique using Multi-resolution Singular Value Decomposition," Defence Science Journal, vol. 61, pp. 479-484, 2011.

[11] B. K. Shreyamsha Kumar, "Multifocus and multispectral image fusion based on pixel significance using discrete cosine harmonic wavelet transform," Signal, Image and Video Processing, vol.7, pp.1125-1143, 2013.

[12] H. Li, S. Wei, and Y. Chai, "Multifocus image fusion scheme based on feature contrast in the lifting stationary wavelet domain," EURASIP Journal on Advances in Signal Processing, pp. 1-16, 2012.

[13] Z. Yuelin, L. Xiaoqiang, and T. Wang, "Visible and Infrared Image Fusion using the Lifting Wavelet," Telecommunication Computing Electronics and Control (TELKOMNIKA), vol.11, No.11, pp. 6290-6295, 2013.

[14] J. Pujar and R.R. Itkarkar, "Image Fusion Using Double Density Discrete Wavelet Transform," International Journal of Computer Science and Network, vol. 5, pp.6-10, 2016.

[15] J. Liu, J. Yang and B. Li, "Multi-focus Image Fusion by SML in the Shearlet Subbands," TELKOMNIKA Indonesian Journal of Electrical Engineering, vol.12, No.1, pp. 618 - 626, 2014.

[16] I.W. Selesnick, RG. Baraniuk, and NG. Kingsbury, "The dual-tree complex wavelet transform," IEEE Signal Processing Magazine, vol.22, pp.123-151, 2005.

[17] W. Zhou, AC. Bovic,HR. Sheikh, and P. Simoncelli, "Image quality assessment: from error visibility to structural similarity," IEEE Transactions on Image Processing, vol. 13, pp. 600-612, 2004. 


\section{BIOGRAPHIES OF AUTHORS}

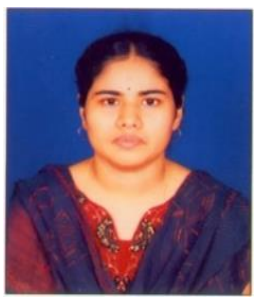

N Radha did AMIE from Institution of Engineers (India), Kolkata and Master of Technology from Jawaharlal Nehru Technological University, Kakinada. She is currently pursuing Ph.D. in Department of Electronics and Communication Engineering, Nagarjuna University, Guntur, Andhra Pradesh. Her main research work focuses on Digital image processing. She is presently working as an Associate professor in the department of Electronics and Communication Engineering in Aditya Engineering College, Surampalem.

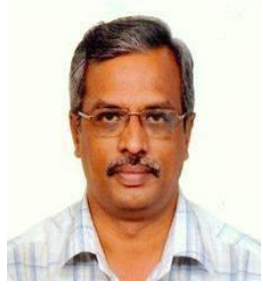

Tummala Ranga Babu obtained his Ph.D. in Electronics and Communication Engineering from JNTUH, Hyderabad, M. Tech in Electronics \& Communication Engineering (Digital Electronics \& Communication Systems) from JNTU College of Engineering (Autonomous), Anantapur, M.S. (Electronics \& Control Engineering) from BITS, Pilani and B.E. (Electronics and Communication Engineering) from AMA College of Engineering (Affiliated to University of Madras). He is currently working as Professor and Head of Department of Electronics \& Communication Engineering in RVR \& JC College of Engineering (Autonomous). His research interests include Image Processing, Embedded Systems, Pattern Recognition, and Digital Communication. 